\title{
REVISITING THE PAPER ON "APPLICATIONS OF GRAPH THEORY TO ENZYME KINETICS AND PROTEIN FOLDING KINETICS: STEADY AND NON-STEADY STATE SYSTEMS"
}

\section{Kuo-Chen Chou}

Gordon Life Science Institute, Boston, Massachusetts 02478, United States of America; kcchou@gordonlifescience.org or kcchou38@gmail.com

About 30 years ago a very important paper on "Applications of graph theory to enzyme kinetics and protein folding kinetics: steady and non-steady state systems" was published [1].

Ever since then, a series of papers for graph theory to study enzyme kinetics [2-8] and protein folding kinetics [9-15].

The graph approach can provide an intuitive feeling for analyzing very complicated biological systems, as clearly demonstrated in the eight masterpieces papers [16-23] by the then Chairman of Nobel Prize Committee.

\section{REFERENCES}

[1] K.C. Chou, Review: Applications of graph theory to enzyme kinetics and protein folding kinetics. Steady and nonsteady state systems, Biophysical Chemistry, 35 (1990) 1-24.

[2] I.W. Althaus, J.J. Chou, A.J. Gonzales, M.R. Diebel, K.C. Chou, F.J. Kezdy, D.L. Romero, P.A. Aristoff, W.G. Tarpley, F. Reusser, Steady-state kinetic studies with the non-nucleoside HIV-1 reverse transcriptase inhibitor U-87201E, J. Biol. Chem., 268 (1993) 6119-6124.

[3] I.W. Althaus, J.J. Chou, A.J. Gonzales, M.R. Diebel, K.C. Chou, F.J. Kezdy, D.L. Romero, P.A. Aristoff, W.G. Tarpley, F. Reusser, Kinetic studies with the nonnucleoside HIV-1 reverse transcriptase inhibitor U-88204E, Biochemistry, 32 (1993) 6548-6554.

[4] I.W. Althaus, A.J. Gonzales, J.J. Chou, M.R. Diebel, K.C. Chou, F.J. Kezdy, D.L. Romero, P.A. Aristoff, W.G. Tarpley, F. Reusser, The quinoline U-78036 is a potent inhibitor of HIV-1 reverse transcriptase, J. Biol. Chem., 268 (1993) 14875-14880.

[5] I.W. Althaus, J.J. Chou, A.J. Gonzales, M.R. Diebel, K.C. Chou, F.J. Kezdy, D.L. Romero, P.A. Aristoff, W.G. Tarpley, F. Reusser, Steady-state kinetic studies with the polysulfonate U-9843, an HIV reverse transcriptase inhibitor, Cellular and Molecular Life Science (Experientia), 50 (1994) 23-28.

[6] I.W. Althaus, J.J. Chou, A.J. Gonzales, M.R. Diebel, K.C. Chou, F.J. Kezdy, D.L. Romero, R.C. Thomas, P.A. Aristoff, W.G. Tarpley, F. Reusser, Kinetic studies with the non-nucleoside human immunodeficiency virus type-1 reverse transcriptase inhibitor U-90152e, Biochem. Pharmacol., 47 (1994) 2017-2028. 
[7] K.C. Chou, F.J. Kezdy, F. Reusser, Review: Kinetics of processive nucleic acid polymerases and nucleases, Anal. Biochem., 221 (1994) 217-230.

[8] I.W. Althaus, K.C. Chou, K.M. Franks, M.R. Diebel, F.J. Kezdy, D.L. Romero, R.C. Thomas, P.A. Aristoff, W.G. Tarpley, F. Reusser, The benzylthio-pyrididine U-31,355, a potent inhibitor of $\mathrm{HIV}-1$ reverse transcriptase, Biochem. Pharmacol., 51 (1996) 743-750.

[9] L. Carlacci, K.C. Chou, Energetic approach to the folding of four a-helices connected sequentially, Protein Eng., 3 (1990) 509-514.

[10] K.C. Chou, L. Carlacci, Energetic approach to the folding of alpha/beta barrels, Proteins: Struct., Funct., Genet., 9 (1991) 280-295.

[11] K.C. Chou, Mini Review: Prediction of protein folding types from amino acid composition by correlation angles, Amino Acids, 6 (1993) 231-246.

[12] K.C. Chou, Graphic rule for nonsteady-state enzyme kinetics and protein folding kinetics, Journal of Mathematical Chemistry, 12 (1993) 97-108.

[13] K.C. Chou, C.T. Zhang, A new approach to predicting protein folding types, J. Protein Chem., 12 (1993) 169-178.

[14] K.C. Chou, C.T. Zhang, Predicting protein folding types by distance functions that make allowances for amino acid interactions, J. Biol. Chem., 269 (1994) 22014-22020.

[15] K.C. Chou, Does the folding type of a protein depend on its amino acid composition?, FEBS Lett., 363 (1995) 127-131. [16] K.C. Chou, S. Forsen, Diffusioncontrolled effects in reversible enzymatic fast reaction system: Critical spherical shell and proximity rate constants, Biophysical Chemistry, 12 (1980) 255-263.
[17] K.C. Chou, S. Forsen, Graphical rules for enzyme-catalyzed rate laws, Biochem. J., 187 (1980) 829-835.

[18] K.C. Chou, S. Forsen, G.Q. Zhou, Three schematic rules for deriving apparent rate constants, Chemica Scripta, 16 (1980) 109113.

[19] K.C. Chou, T.T. Li, S. Forsen, The critical spherical shell in enzymatic fast reaction systems, Biophysical Chemistry, 12 (1980) 265-269.

[20] T.T. Li, K.C. Chou, S. Forsen, The flow of substrate molecules in fast enzymecatalyzed reaction systems, Chemica Scripta, 16 (1980) 192-196.

[21] K.C. Chou, R.E. Carter, S. Forsen, A new graphical method for deriving rate equations for complicated mechanisms, Chemica Scripta, 18 (1981) 82-86.

[22] K.C. Chou, N.Y. Chen, S. Forsen, The biological functions of low-frequency phonons: 2. Cooperative effects, Chemica Scripta, 18 (1981) 126-132.

[23] K.C. Chou, S. Forsen, Graphical rules of steady-state reaction systems, Can. J. Chem., 59 (1981) 737-755. 